\title{
Compartmentalization - A Prerequisite for Maintaining and Changing an Identity
}

\author{
Philipp Rottmann", Thomas Ward ${ }^{\mathrm{b}}$, and Sven Panke ${ }^{*_{a}}$
}

\begin{abstract}
The chemical manipulation of DNA is much more convenient than the manipulation of the bioproducts, such as enzymes, that it encodes. The optimization of bioproducts requires cycles of diversification of DNA followed by read-out of the information into the bioproduct. Maintaining the link between the information - the genotype - and the properties of the bioproduct - the phenotype - through some form of compartmentalization is therefore an essential aspect in directed evolution. While the ideal compartment is a biological cell, many projects involving more radical changes in the bioproduct, such as the introduction of novel cofactors, may not be suitable for expression of the information in cells, and alternative in vitro methods have to be applied. Consequently, the possibility to produce simple and advanced micro compartments at high rates and to combine them with the ability to translate the information into proteins represents a unique opportunity to explore demanding enzyme engineering projects that require the evaluation of at least hundreds of thousands of enzyme variants over multiple generations.
\end{abstract}

Keywords: Compartmentalization · Enzyme engineering · High-throughput screening · Microfluidics · Water-in-oil emulsion

\section{Introduction}

The biological cell is a perfect example of advanced compartmentalization strategies. Compartmentalization helps to organize complex processes, in the course of which information is converted into biomaterials (nucleus, cytoplasm, and endoplasmic reticulum in eukaryotic cells), energy is converted (mitochondria), reactions are catalyzed (membrane synthesis), proteins are degraded (lysosomes), or the spread of toxic intermediates is prevented (bacterial micro compartments) to name only a few examples. ${ }^{[1]}$

In most cases it is not only the physical separation between two environments but also the embedded membrane proteins that are responsible for specific transport between compartments, catalysis of compounds and signaling processes. Consequently, artificially produced vesicles having at least one lipid bilayer, so called liposomes, have been used for a long

\footnotetext{
${ }^{\star}$ Correspondence: Prof. Dr. S. Panke

E-Mail: sven.panke@bsse.ethz.ch

aDepartment of Biosystems Science and Engineering

ETH Zurich, Mattenstrasse 26

$\mathrm{CH}-4058$ Basel

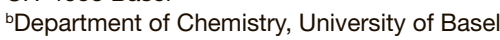

time as an important method to study lipid properties and behaviors, and membrane protein functionality in vitro, and several examples for this approach can be found in this issue (see Lim and coworkers ${ }^{[2]}$ ). Low stability of these artificially produced liposomes triggered, among other things, the development of polymer molecules mimicking a lipid membrane. Such polymersomes have been used to encapsulate active enzymes and integrate membrane proteins in the polymer shell and they exhibit, in general, higher stability compared to liposomes and are therefore of particular interest for drug delivery, ${ }^{[3]}$ see the article by Palivan and coworkers in this issue. ${ }^{[4]}$ Another important application for artificially-produced compartments is the creation of miniaturized reaction chambers for a directed evolution process to endow whole organisms ${ }^{[5]}$ or single enzymes ${ }^{[6]}$ with new properties such as novel substrate specificity or improved yield on starting material. In particular in the latter field, artificial compartments allow the exploration of new avenues to endow enzymes with novel properties, as they allow the analysis of large numbers of enzyme variants in conditions that can be quite distinct from the natural environment of enzymes with respect to aspects such as $\mathrm{pH}$, temperature, and liquid phase composition, so they cannot be analyzed in cells. Here, we will give a brief overview about past and present compartmentalization technologies for enzyme engineering. Additionally, some examples will be presented of directed evolution approaches that were conducted in in vitro produced compartments.

\section{Production Techniques for Different Compartments}

Different in vitro compartmentalization techniques are available today but most of them combine the same principle of dispersing an aqueous solution into a waterimmiscible phase to produce a water-inoil (w/o) emulsion. The water-immiscible phase can consist of a mixture of different lipids to form liposomes which are more likely to behave like an original biological membrane than other materials. ${ }^{[7]}$ Alternatively, chemically synthesized block-copolymers can be used, which are not only able to form reaction compartments but can also exhibit membrane-like functionality such as the capability to integrate functional membrane proteins for selective transport. ${ }^{[8]}$ Such polymersomes are also obtained by mixing the polymers with an aqueous phase. Finally, different oil types can be used to generate containers as w/o emulsions. Such emulsions need to be stabilized by surfactant molecules to prevent coalescence and usually do not exhibit sophisticated membrane functions and can thus only be seen as highly individualized separated reaction chambers. Different protocols are available for the production of liposomes, ${ }^{[9]}$ polymersomes ${ }^{[8]}$ or water-in-oil emulsions, depending on the application. Because of their important role in enzyme engineering, we will, in the following, focus on the production of w/o emulsions and their specific applications.

Two simple categories of emulsions can be distinguished depending on the width of the distribution of compartment diameters. 
The traditional way of dispersing water into an oil phase by stirring or vigorous shaking leads to emulsions with a polydisperse droplet population, i.e. a population with broadly different diameters (Fig. 1a). By contrast, the more controlled production conditions in microfluidic chips lead to monodisperse droplet populations characterized by a rather small distribution in diameter (Fig. 1b). In the following, these two characteristics and their production procedures are described in more detail. Many insights into the fundamentals of the field of emulsion production and stabilization were obtained in the chemical and food industry for the production of margarine (w/o emulsion) and the cosmetic industry for the production of creams (oilin-water or $\mathrm{o} / \mathrm{w}$ emulsion). The great potential of w/o emulsions as reaction compartments for biological methods such as a directed evolution screen system was initially explored by Tawfik and Griffiths. ${ }^{[10]}$ The emulsions were produced by mixing an aqueous solution in mineral oil and surfactant with a stirring bar, and the aqueous phase contained a DNA molecule that contained the information encoding the enzyme variant and a cell-free extract, which in turn contained the entire machinery for protein production, including ribosomes, tRNA, etc. The produced w/o droplets were highly polydisperse and contained droplets with diameters between 1 and $8 \mu \mathrm{m}$ (volumes between 0.5 and $270 \mathrm{fL}$ ) and allowed compartmentalized expression of the unique information in each droplet. In this case, they enriched a methyltransferase from an excess of $10^{7}$ variants without methyltransferase activity. This enrichment is facilitated by the fact that the methyltransferase methylates a specific DNA sequence rendering it insensitive to DNA restriction digest thereby specifically digesting only the DNA of unwanted variants Furthermore, a similar approach was used to evolve a DNA polymerase that showed higher tolerance against inhibitor substances ${ }^{[11]}$ or the incorporation of hydrophobic base analogues. ${ }^{[12]}$ Clearly, applications other than DNA modifying enzymes required different read-out methods. If, for example, directed evolution of an enzyme is desired that converts a non DNA substrate, a different assay needs to be established. Detection can be achieved by using a non-fluorescent substrate that upon catalysis turns into a fluorescent product, so that enzyme activity in small droplets can in principle be analyzed via flow cytometry. However, standard fluorescent activated cell sorters (FACS) cannot deal with w/o droplets, because sorting depends on charging an aqueous droplet surface. This becomes possible when applying water-in-oil-in-water (w/o/w) double emulsions, which can be produced by mixing the first w/o emulsion into a second aqueous phase with a stirring bar or by vigorously shaking using a vortex mixer. ${ }^{[13]}$ Such double emulsions can be subsequently analyzed with a FACS device and containers with active enzymes that generate a fluorescent signal can be sorted with rates of up to 25,000 events $\mathrm{s}^{-1}$. With this set-up, the catalytic activity of various enzymes such as $\beta$-galactosidase, ${ }^{[14]}$ phosphotriesterase ${ }^{[15]}$ and thiolactonase ${ }^{[16]}$ could be improved. The typical diameter of droplets in the double emulsions mentioned above ranged from $10-30 \mu \mathrm{m}$ with volumes between $0.5-15 \mathrm{pL}$.

This broad volume distribution leads to drastic product concentration differences for enzymes even if they display similar specific activity and thus limits the usefulness. However, circumventing polydispersity and increasing control over the production of the emulsions required a new technology. The advent of microfluidic techniques was then used to precisely control the generation of droplets, but it is associated with the disadvantage of reduced droplet-formation rate. The production of droplets via stirring or vortexing can lead to the formation of $10^{11}$ droplets within a few seconds. With microfluidic devices, only approximately $10^{8}$ droplets can be realistically prepared in two hours, depending on the chip geometry. Of course, also at such high rates, compartmentalization is still an excellent tool for enzyme evolution. W/o emulsions with monodisperse droplet population can be produced either in poly(dimethylsiloxane) PDMS chips or a glass capillary set-up. With the two-dimensional PDMS chip, the oil stream emulsifies an aqueous stream at a Y-junction. ${ }^{[17]}$ In a three-dimensional glass capillary setup an oil sheath fluid emulsifies a water stream. ${ }^{[18]}$ To guarantee a constant flow and reliable production of droplets the surfaces of the glass capillary or the PDMS chip need to be treated in a specific way to prevent wetting. Wetting is the ability of liquids to hold contact to a solid surface and depends on the properties of the surface. Production of w/o droplets thus requires a hydrophobic treatment of the PDMS channels or glass capillaries. The production rate of the droplets is usually in the $\mathrm{kHz}$ range but it was already reported to advance to the low $\mathrm{MHz}$ range, coming close to the number of droplets that can be produced with the traditional techniques mentioned above. ${ }^{[19]}$ The commonly used oil phase for the production of these reaction vessels is composed of fluorinated oil and fluorinated surfactants for stability. If an optical read-out is needed, then FACS cannot be used for the aforementioned reasons. Instead, on-chip fluorescent read-out and sorting was developed and sorting frequencies of up to $2,000 \mathrm{~s}^{-1}$ can be reached, which is still below the frequencies that can be reached with a standard FACS device. Nevertheless, with this technology, promiscuous enzymes for the catalysis of phosphotriesterases and sulfotriesterases were found in a metagenomic library after the screening of $3 \times 10^{7}$ clones. ${ }^{[20]}$ If higher
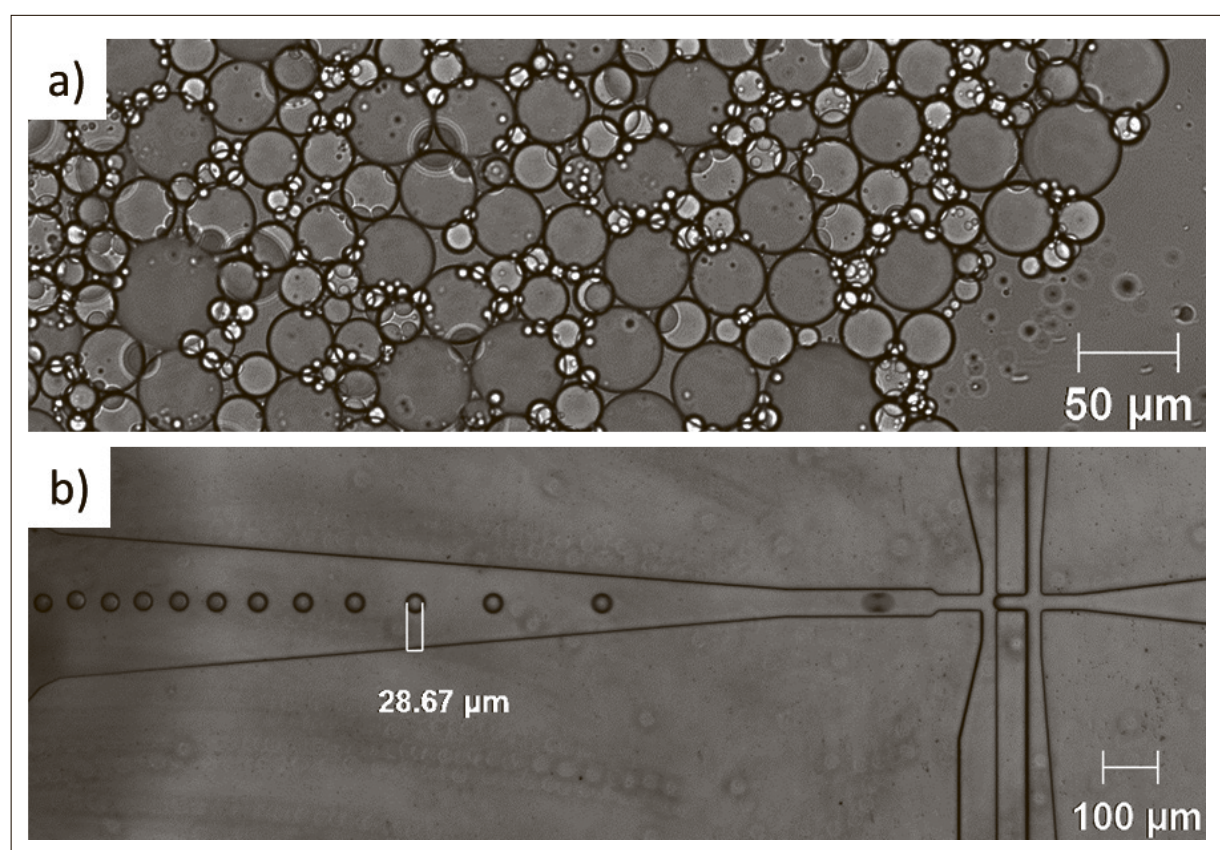

Fig. 1. Poly- and monodisperse water-in-oil (w/o) emulsion. a) An aqueous solution was mixed with an excess of oil (1:10) and vortexed for $10 \mathrm{sec}$. The oil phase contained HFE7500 and 3\% Krytox as surfactant for droplet stabilization. The formed droplets with different diameters from 2 to $50 \mu \mathrm{m}$. b) A poly(dimethylsiloxane) (PDMS) microfluidic chip with two aqueous inlets and one oil inlet (HFE7500 with 3\% Krytox) was used to produce monodisperse droplets with a diameter of $28 \mu \mathrm{m}$. 
sorting rates than $2,000 \mathrm{~s}^{-1}-$ as can be obtained by a standard FACS - are required, then a second aqueous phase is needed. This can be achieved again with either PDMS chips or a glass capillary set-up. Here, two emulsions need to be combined, which requires a more complex chip geometry. First, the aqueous stream is emulsified by the oil stream, and correspondingly the following channels need to have a hydrophobic surface. At a second junction the w/o stream is re-emulsified into an aqueous stream, the subsequent channels need to have a hydrophilic surface. Treatment of one microfluidic chip with two different surface coatings for the production of double emulsions is difficult to achieve due to possible crosstalk between the channels. Separating the microfluidic chips into two stages, one for the generation of the w/o emulsion and one for the generation of the w/o/w emulsion prevents such difficult channel surface treatments. This approach also allows the first emulsion to be collected after formation in the first chip, incubation of droplets off chip, and later re-injecting into the second, hydrophilic chip. ${ }^{[21]}$

\section{Applications of Artificially Produced Compartments}

The applications for small compartments in life sciences are manifold and range from enzyme ${ }^{22]}$ and ribozyme engineering[23] via analysis of DNA populations ${ }^{[24]}$ to genome sequencing based on next-generation sequencing technology. ${ }^{[25]}$ The applications can be divided into two parts, in vitro and in vivo screenings. A typical in vivo screening approach consists of four major steps starting with gene diversification to generate a DNA library, ${ }^{[26]}$ using the library to transform a host organism such as E. coli and subsequent expression of the information, screening and sorting of the positive clones harboring a promising variant, recovery of the genotype and either further diversification for a next round or analysis via sequencing. In the in vitro approach, the DNA library is directly emulsified with the suitable components required for reading out the information (e.g. cell-free extract, see above), and thus the sometimes limiting host transformation step can be omitted. Depending on the application, this approach allows screening for a suitable phenotype on the DNA, RNA and protein level.

\section{Applications in vivo}

\section{Cell Lysis}

Despite the possibly misleading terminology, many in vivo applications in micro compartments ultimately require the release of a protein from the cell that contains the gene and was used to read out the information, followed by the reaction that generates the selectable signal (Fig. 2a). Positive candidates can be selected and retrieved in the form of DNA from the droplet, amplified and undergo a next round of selection or can be analyzed via sequencing. [27]

\section{Reactions Outside of a Cell}

One of the advantages of compartments is that for reactions taking place outside of cells but are encoded inside of them, the linkage between genotype and phenotype is still maintained, as it is the case for an example with secreted enzymes. Coencapsulation of the substrate leads to the formation of the product that is physically retained in the vicinity of the gene-carrying cell and positive clones. The droplets can be sorted (Fig. 2b) and either the DNA is retrieved via colony PCR or if the cell (or cells) in the compartment is still alive, the cells can be re-grown, for example on solid media. ${ }^{28]}$ Alternatively, the enzyme is not secreted into the medium but retained on the outer surface of the cell, a technique called protein- or enzyme display. The phenotype-determining reaction still takes place on the outside of the cell (Fig. 2c). This was successfully demonstrated with the improvement of horseradish peroxidase (HRP) which was displayed on the surface of a yeast cell. ${ }^{[29]}$ The evolved peroxidase showed a 10-fold increase of activity against a specific substrate compared to its wild type.

\section{Applications in vitro}

\section{Cell-free Protein Synthesis}

The first compartments used for enzyme engineering by Tawfik and Griffiths contained only one copy of a diversified gene. To produce the protein, the HaeIII methyltransferase, they used cell-free protein synthesis (see above). ${ }^{[30]}$ This technique alleviates the step of ligating the gene into a plasmid and using the resulting library for the transformation of an organism (Fig. 2d). Furthermore, proteins that are toxic or harm the growth of the organism can still be expressed and improved

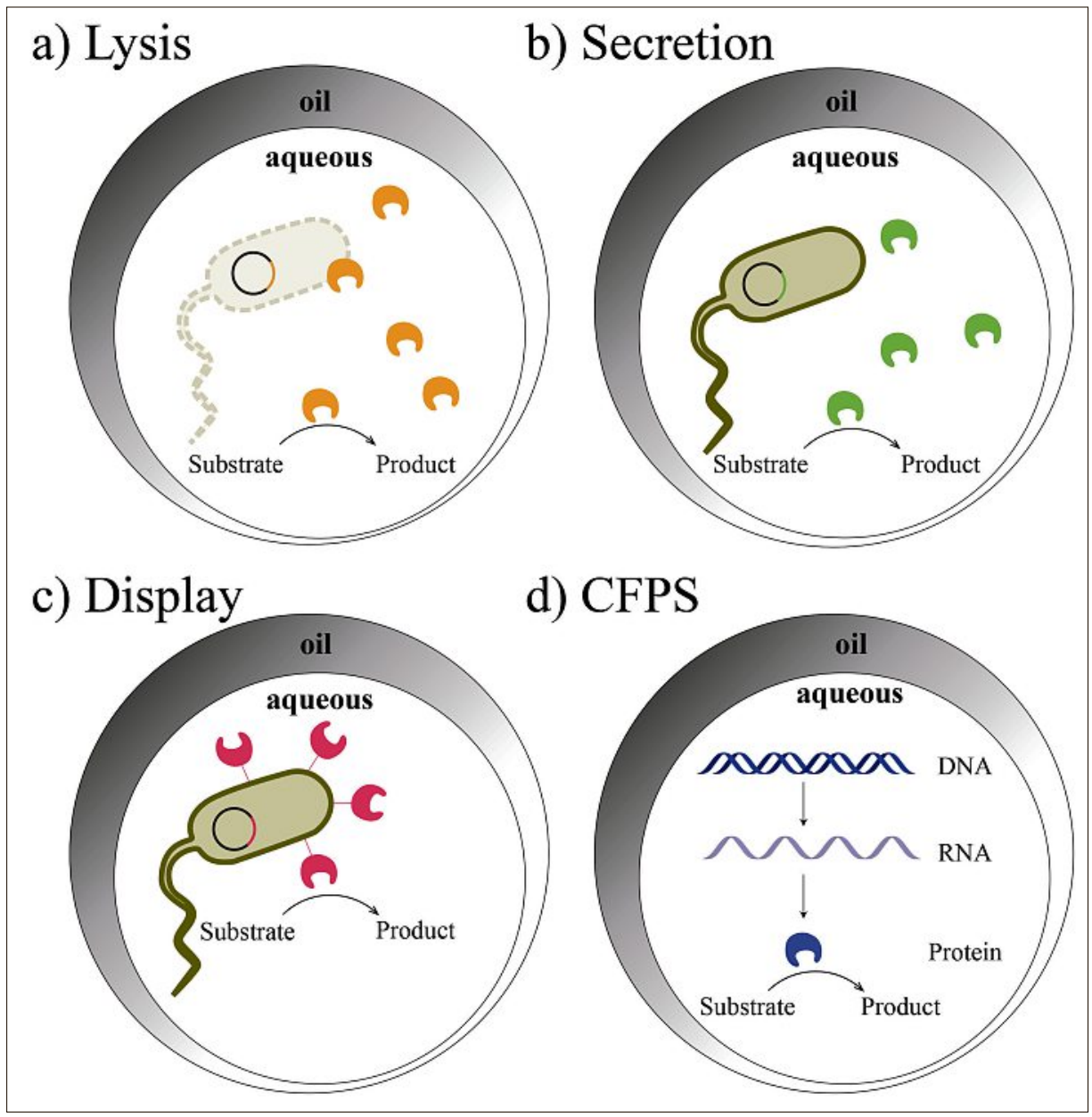

Fig. 2. Simple representation of expression systems that can be used in combination with compartmentalization techniques. ${ }^{[30]}$ a) Expression of a specific gene product in a cell and subsequent lysis after encapsulation. b) Secretion of the gene product after encapsulation of the host organism. c) Cell-surface display of the gene product after encapsulation of the organism. d) Cell-free protein synthesis of the encapsulated gene. 
variants can be sorted out. With this technique, the substrate already needs to be present during the synthesis of the gene. A drawback is the low amount of DNA that is present per droplet after compartmentalization. As each droplet is to have only one information variant, at the beginning each compartment must contain only one DNA molecule. This in turn typically leads to low amounts of protein produced and potential variants easily get lost during the sorting process. A suitable strategy is to include a DNA amplification step at some point before compartment formation, e.g. by PCR. The involved temperature increase would lead to an inactivation of the capacity to read out the information into protein, therefore, the amplification step needs to be kept separate from the information read-out. This can be achieved in microfluidic settings by injecting a cell-free protein synthesis mixture into each droplet after amplification. Positive variants can be sorted out based on a robust signal and the DNA retrieved. ${ }^{[31]}$

\section{RNA Screening}

Not only enzymes can be the target of directed evolution but also RNA molecules, which find applications for their capacity to selectively bind small molecules (e.g. as riboswitches) or to catalyze reactions (ribozymes).[23] A sophisticated variation of the protocol includes the use of droplets in a transcriptomics application: mRNA molecules containing the poly-adenosine tail can be captured via poly-thymidine functionalized micro beads from single cells encapsulated into droplets, reverse transcribed and amplified for further single cell transcriptome analysis. ${ }^{[32]}$

\section{Magnetic Beads}

Magnetic beads can serve as an anchor point for DNA molecules much as a cell gives a first compartment to a bacterial chromosome or plasmids. The beads have the advantage that they can be easily separated from an emulsion with an externally placed magnet. Target genes can be amplified ${ }^{[33]}$ on the beads and expressed via cell-free protein synthesis. ${ }^{[34]}$ Several techniques exist to afterwards bind the protein to its specific DNA on the magnetic bead to generate a covalent genotype-phenotype linkage. ${ }^{[35]}$ Placing the populated magnetic bead then in a compartment in an w/o or a w/o/w emulsion allows for the use of this technique for enzyme evolution as the reaction product remains in the same compartment as the information on the bead - although also alternative linkage schemes are possible if a fluorescent reaction product can be bound to the magnetic bead directly.

\section{Outlook}

Even though compartments were presented here in the context of biocatalyst evolution, they are in fact rather sophisticated reaction systems with a hint of the basics for minimal life. Especially, the cell-free protein synthesis approach captures already quite a large number of functions of a minimal cell. ${ }^{[36]}$ While neither compartments produced with fluorinated oil nor with polymersomes have an equivalent in known biological systems, they illustrate directions into which the field is developing: compartments become, on the one hand, more sophisticated and, on the other hand, address specific deficiencies of biological or biology-derived systems (such as limited stability) with molecules derived from chemical synthesis (e.g. block-copolymers). It will be interesting to see whether proteins can be adapted to operate in such artificial systems, in particular in the corresponding membranes or whether they need to be evolved to do so. Ultimately, the reaction compartments will become increasingly more functionally sophisticated, e.g. by the integration of membrane proteins to improve communication with the exterior of the compartment - and thus become more and more like a re-engineered version of a cell.

\section{Acknowledgements}

This work is supported by the Department of Biosystems Science and Engineering and the Swiss National Science Foundation as part of the NCCR in Molecular Systems Engineering.

Received: May 1, 2016

[1] C. A. Kerfeld, S. Heinhorst, G. C. Cannon, Annu. Rev. Microbiol. 2010, 64, 391.

[2] S. Vujica, C. Zelmer, R. Panatala, R. Y. H. Lim, Chimia 2016, 70, 413.

[3] K. Langowska, J. Kowal, C. G. Palivan, W. Meier, J. Mater. Chem. B 2014, 2, 4684.

[4] M. Garni, T. Einfalt, M. Lomora, A. Car, W. Meier, C. G. Plaivan, Chimia 2016, 70, 424.

[5] A. Meyer, R. Pellaux, S. Potot, K. Becker, H. P. Hohmann, S. Panke, M. Held, Nat. Chem. 2015, 7, 673 .

[6] D. S. Tawfik, A. D. Griffiths, Nat. Biotechnol. 1998, 16, 652 .

[7] P. Walde, S. Ichikawa, Biomol. Engin. 2001, 18, 143.

[8] P. Tanner, P. Baumann, R. Enea, O. Onaca, C. Palivan, W. Meier, Acc. Chem. Res. 2011, 44, 1039.

[9] B. Maherani, E. Arab-Tehrany, M. R. Mozafari, C. Gaiani, M. Linder, Curr. Nanosci. 2011, 7 , 436.
[10] A. D. Griffiths, D. S. Tawfik, Curr. Opin. Biotechnol. 2000, 11, 338 .

[11] F. J. Ghadessy, J. L. Ong, P. Holliger, Proc. Natl. Acad. Sci. USA 2001, $98,4552$.

[12] D. Loakes, J. Gallego, V. B. Pinheiro, E. T Kool, P. Holliger, J. Am. Chem. Soc. 2009, 131, 14827.

[13] K. Bernath, M. Hai, E. Mastrobattista, A. D. Griffiths, S. Magdassi, D. S. Tawfik, Anal. Biochem. 2004, 325, 151

[14] E. Mastrobattista, V. Taly, E. Chanudet, P. Treacy, B. T. Kelly, A. D. Griffiths, Chem. Biol. 2005, 12, 1291.

[15] R. D. Gupta, M. Goldsmith, Y. Ashani, Y. Simo, G. Mullokandov, H. Bar, M. Ben-David, H. Leader, R. Margalit, I. Silman, J. L. Sussman, D. S. Tawfik, Nat. Chem. Biol. 2011, 7, 120.

[16] A. Aharoni, G. Amitai, K. Bernath, S. Magdassi, D. S. Tawfik, Chem. Biol. 2005, 12, 1281.

[17] J. C. McDonald, D. C. Duffy, J. R. Anderson, D. T. Chiu, H. K. Wu, O. J. A. Schueller, G. M. Whitesides, Electrophoresis 2000, 21, 27.

[18] L. L. A. Adams, T. E. Kodger, S. H. Kim, H. C. Shum, T. Franke, D. A. Weitz, Soft Matter 2012, 8, 10719.

[19] J. U. Shim, R. T. Ranasinghe, C. A. Smith, S. M. Ibrahim, F. Hollfelder, W. T. Huck, D. Klenerman, C. Abell, ACS Nano 2013, 7, 5955.

[20] P. Y Colin, B. Kintses, F. Gielen, C. M. Miton, G. Fischer, M. F. Mohamed, M. Hyvonen, D. P. Morgavi, D. B. Janssen, F. Hollfelder, Nat Commun. 2015, 6, 10008.

[21] A. Zinchenko, S. R. Devenish, B. Kintses, P. Y. Colin, M. Fischlechner, F. Hollfelder, Anal. Chem. 2014, 86, 2526

[22] M. Levy, A. D. Ellington, Chem. Biol. 2008, 15, 979.

[23] M. Ryckelynck, S. Baudrey, C. Rick, A. Marin, F. Coldren, E. Westhof, A. D. Griffiths, $R N A$ 2015, 21, 458.

[24] F. Diehl, M. Li, Y. He, K. W. Kinzler, B Vogelstein, D. Dressman, Nat. Meth. 2006, 3 , 551.

[25] J. M. Rothberg, J. H. Leamon, Nat. Biotechnol. 2008, 26, 1117.

[26] E. Henke, U. T. Bornscheuer, Biol. Chem. 1999 $380,1029$.

[27] B. Kintses, C. Hein, M. F. Mohamed, M Fischlechner, F. Courtois, C. Leine, F. Hollfelder, Chem. Biol. 2012, 19, 1001.

[28] B. L. Wang, A. Ghaderi, H. Zhou, J. Agresti, D. A. Weitz, G. R. Fink, G. Stephanopoulos, Nat. Biotechnol. 2014, 32, 473.

[29] J. J. Agresti, E. Antipov, A. R. Abate, K. Ahn, A. C. Rowat, J. C. Baret, M. Marquez, A. M. Klibanov, A. D. Griffiths, D. A. Weitz, Proc. Natl. Acad. Sci. USA 2010, 107, 4004.

[30] P. Y. Colin, A. Zinchenko, F. Hollfelder, Curr. Opin. Struct. Biol. 2015, 33, 42.

[31] A. Fallah-Araghi, J. C. Baret, M. Ryckelynck, A. D. Griffiths, Lab on a Chip 2012, 12, 882.

[32] E. Z. Macosko, A. Basu, R. Satija, J. Nemesh, K. Shekhar, M. Goldman, I. Tirosh, A. R. Bialas, N. Kamitaki, E. M. Martersteck, J. J. Trombetta, D. A. Weitz, J. R. Sanes, A. K Shalek, A. Regev, S. A. McCarroll, Cell 2015 161, 1202.

[33] D. Dressman, H. Yan, G. Traverso, K. W Kinzler, B. Vogelstein, Proc. Natl. Acad. Sci. USA 2003, 100, 8817 .

[34] B. Zhu, T. Mizoguchi, T. Kojima, H. Nakano, Plos One 2015, 10, e0127479.

[35] L. Diamante, P. Gatti-Lafranconi, Y. Schaerli, F. Hollfelder, PEDS 2013, 26, 713.

[36] R. Gil, F. J. Silva, J. Pereto, A. Moya, $M M B R$ 2004, 68, 518 